\title{
The Treatment of Habitual Dysphonia
}

Joan Hi. van Thal, M.B.E., F.C.S.T.

From time to time patients consult a laryngologist complaining of hoarseness and loss of voice for which no cause can be found either in the larynx or its nerve supply. Indirect laryngoscopy commonly shows a deficiency in the approximation of the vocal folds, occasionally interference by the ventricular bands during phonation and various other laryngeal dysfunctions.

By "loss of voice" they may mean a variety of things, but hardly ever real aphonia; loss of carrying power of voice, restriction of range of pitch, changes in its quality such as huskiness, cracking or harshness all enter into the picture, which is too varied to be described in all its kaleidoscopic manifestations.

The patients in question are either professional voice users (in which category one should include amateur performers, and people who do a lot of talking in the course of their work, such as interviewing, telephoning etc.) who find their voices no longer adequate to meet the demands made on them, or people who have become justifiably alarmed about their chronic hoarseness and wisely consult a laryngologist, and in both instances are reassured about their condition, told it is due to habitual misuse of the voice and referred to the speech therapist.

Much now depends on the importance which the patient attaches to the quality of the voice whether they make use of the services of the speech therapist, available to them free of charge under the National Health Service, so that the question of expense does not enter into it. The decision may depend on common sense, but it may also be influenced by a psychological overlay of the dysphonia; thus refusal may be due to difficulty in getting time off from work for someone not a professional voice user, or to an unconscious desire to retain the symptom which is of some value to the patient whose dysphonia is of a psychogenic nature.

Similarly agreement to have treatment may be on a rational basis, the necessity to recover the voice for professional or social reasons, or it may be for the sake of being interesting and satisfying a subconscious urge for attention.

Those who refuse voice therapy are of no further interest; but a careful assessment of the reason for the vocal dysfunction must be made in those who seek rehabilitation. The situation may be the uncomplicated one of a teacher, public speaker or the like on whose voice special demands have been made without their having been trained to meet these requirements; it may be a singer who has been badly taught, or who has misunderstood his instructor's directions and explana- tions. These people are in a category where corrective work is required, and this work is on the borders of normal voice training and "voice therapy": a routine as set out below can be followed.

Psychogenic dysfunctions can approximate closely to hysterical aphonia - an unconscious desire to shake off responsibilities, e.g. a headmaster who had; suffered from laryngitis and been unable for that reason to address the end of term assembly began to have recurring bouts of loss of voice - he carried on with his work with enthusiasm except if addressing the assembled school. (Given insight into his recurring dysphonia he recovered completely with a minimum of direct voice training).

In many instances however there is no hysterical element in the voice change, but it is expressive of a state of mind of the patient. Normally the voice expresses our feeling; thus in "people in quandaries" of a persistent nature the harsh and cracked, feeble and husky, low constricted or high pitched strident sound reflects their emotional condition, e.g. a harassed housewife who had suffered much grief and worry over a period of two or three years, torn between her duty to a widowed mother and to her husband, her whole posture and facial expression as well as her feeble, croaking voice portrayed her mental stress; indirect laryngoscopy showed that the right vocal fold made a spasmodic abductor movement posteriorly during phonation, though the anterior part and the liv.c. adducted normally. Considerably more in the nature of reassurance and relief from tension by relaxation and freely talking about her troubles was done concurrently with vocal retraining than would be envisaged for cases of vocal dysfunction only due to ignorance of good technique.

It would be equally wrong to assume that there must be psychological complications in these cases as it would be to take the opposite view. Thus, as in all cases referred for speech therapy, a full case history has to be compiled. It is wise, however much of ones time one devotes to this at the first interview, to take some of the preliminary steps in vocal retraining from the start, since these people usually expect that something be done. To affront the patient at this early, stage by too obvious delving would have a very serious affect on rapport, particularly if there are no psychological complications. No harm can be done by doing postural exercises, even if soon after the course of treatment has begun one realizes one is dealing with a psycho-neurosis beyond the scope of the speech therapist, in that case one should hand over to the psycho-therapist and voice therapy is contra-indicated. In the less serious psychogenic cases a combination of counselling and direct voice correction can safely be undertaken. 
The routine of voice correction must include:

1. Postural exercises.

2. Simple breathing exercises.

3. Exercises in projection of the voice.

4. Establishing of good habits of voice production in speaking and reading; for singers - also in song.

\section{POSTURAL EXERCISES}

It is the posture of the head and neck that is of supreme importance; unless the patient has to stand and speak, and must therefore acquire a good stance, it simplifies matters to do these exercises seated. A mirror allowing him to see head and should is essential.

There is invariably misplaced effort, even though where there is failure in adduction of the vocal folds the first impression is the opposite; the deficient adduction may be due to activity of the abductors, contrary to the Law of Reciprocal Innervation, when these should relax.

- Let the patient be seated on a chair that offers good support to the thighs and the back, without tilting the head forward or back:

1. Ensure that the chin is held at the correct angle; commonly it is tilted slightly upwards, sometimes it is pushed forward as well; occasionally, but not very often, the chin is tucked in. Stand behind the patient, take his head between your hands and firmly but gently move it from side to side, down and up in a forward direction, and finish with the head held straight, chin on a horizontal plane. Let the patient bring the back of his fingers, held straight, under the chin to feel the position; care must be taken he does not tilt his hand up to meet the chin if he happens to be holding the chin thus. Gradually he must learn to do without looking in the mirror or feeling the position of the head with his hand, and become aware whether he is holding his head straight wtihout strain, witout such aids.

2. Let the patient bend the head forward and straighten it again, several times running. This to be done without jerks, and at a moderate pace.

3. Negative practice-bend head forward; straighten; crane a little; straighten again. The craning phase must be much shorter than each of the others.

4. Jaw exercises - patient places a hand on either cheek and gently strokes downwards bringing the mandible down and so opening the mouth about an inch. Close again and repeat several times.

Open and close jaw effortlessly without stroking.

(Chewing exercise as recommended by E. Froeschels should also be done.)

\section{BREATHING EXERCISES}

No elaborate breathing exercises need be done, except possibly by singers and those who have to speak in large auditoria.

There is every likelihood, however, that these patients have some unpropitious habits, such as raising the shoulders, over-breathing or keeping the thorax rigid; nearly all will exhale with a fricative sound (not a consonant) which indicates constriction of the pharynx or oral cavity, when they breathe out through the open mouth.

N.B.-Be sure that they open the mouth in the manner practised under jaw exercises, neither too little nor too much; do not allow them to close the mouth till the end of the expiratory phase, unless practising breathing out with formation of speech sounds, such as $\mathrm{m}$.

1. Place hands on sides, just below the sternum, with finger tips meeting. No pressure to be exercised by the hands. Breathe out through nose as a preliminary, fingers will overlap a little. Now breathe in to a count of two, out for two; both through the nose. The hands are there to feel the lateral movement; watch for shoulder heaving, swaying of the torso and other useless extraneous movements.

2. Breathe in through the nose, out through the mouth, silently. Take the exercises at the normal respiratory rate of about 17 to the minute.

3. Slightly prolong the expiratory phase, but leave the inspiratory as before.

4. While breathing out change the position of lips and tongue, as if you were about to whisper "a-u" three or four times running; vary the exercise with "a-i" but still make no sound.

5. Allow the breath to "pour out" audibly without friction, for the same vowel series. "Ha-hu, ha-hi"*

(N.B.-Do not use the word whisper to the patient, for he will certainly start to use a forced whisper if you do. Do not encourage audible exhalation with the articulatory organs static, this is more likely to lead to strain, and these exercises are preparatory to training in voice projection, which is similarly better done on a series of syllables than on a single vowel.)

*A random pattern of vowels may prove satisfactory taken with the chewing technique.

\section{HUMMING}

1. Find a note comfortably about the middle of the patient's range. Let him hum "mmm" softly for two seconds or so, placing a finger on the lips the while to feel the vibrations reaching them.

2. Prolong the humming a little growing louder and 
then allowing the sound to diminish again. Pluck the lip while doing so, and a "twanging" sound should be heard if the voice is well projected, with full nasal resonance. It is possible to miss the twanging in spite of satisfactory resonance if " $n$ " is being sung instead of " $\mathrm{m}$ ", however.)

In the case of singers who have learned a wrong technique it is better to practise on speaking glides at first instead of on a sung note: Similarly there are people who believe they are unable to sing and demur at sung exercises. for whom speaking glides only should be used.

3. Either hüm on a series of singing notes, or on a spoken glide on " $m$ ". For singers gradually increase the range of the notes up and down the scale.

\section{Other Voice Projection Exercises.}

4. Open mouth, as practised before, and sigh out gently on a descending glide. No hard attack (glottal stop) no scraping.

Gradually reduce the sighing element and emit "a" or another vowel on a descending or ascending glide without constriction in any part of the vocal apparatus.

5. Using " $m$ " as initial, practise a series of syllables with the vowels practised during breathing exercises. "ma-mu, ma-mu, ma-mu" "ma-mi, ma-mi, ma-mi" "ma-mu-ma-mi, ma-mu-ma-mi".

6. Proceed to words with similar sound patterns e.g.

\section{English}

May moon

My man

\section{Dutch}

Mijn moe

Mijn man

\section{Sentences}

My mother made some marmalade/ one morning/ last month.
De molenaar maalt nie meer/ voo r Maandag/ maar mijn meel is op.
The common error of constricting the voice at the end of a sentence can be overcome by the following simple device - pretend you are going to say a word or two more but donot say them aloud. In the above practice sentences the patient is told the words up to the second stroke, but instructed only to speak those up to the first stroke "marmalade" or "meer" aloud, and say the rest "in his head". Next tell them the remainder of the sentence, allow them to speak aloud to "morning" or Maandag", but say the rest in their heads.

Finally allow them to speak the whole sentence, but still keep up the pretence that there is a word or two to follow.
7. Practice on words of little importance.

Induce the patient to transfer his technique, so far depending on the propitious initial $\mathrm{m}$, to other words and phrases. In order to avoid any strain that may be set up by an earnest attempt to remember the words of the exercise, it is best at this point to use such devices as counting, repeating the names of the days and months, familiar telephone numbers, conventional phrases of greeting etc.

The device "think ariother word" is to come" can again be used, e.g. count to ten but imagine you are going on to eleven; say "good morning" pretend you were about to say "good morning, sir" and change your mind about "sir".

\section{Reading Aloud.}

This is a useful medium for establishing correct habits, but it has many pitfalls. For those who are not accustomed to reading aloud it is unpropitious, since it causes stress militating against the use of their new technique, and if they never will have to read:aloud in their daily life it is purposeless to practise the art.

For those who are accustomed to read aloud there is the pitfall of an acquired special reading voice, so that such :practice causes relapse, thus it should be postponed till the last stages of rehabilitation of voice.

\section{Speeches.}

These can be more safely introduced at the intermediate stage. The subject matter will depend on the interests of the speaker - ranging from the retelling a film recently seen to an expert short lecture on biochemistry.

\section{Material to learn by heart.}

Particularly where reading aloud is to be avoided and where there is little originality of thought for speeches, it is well to provide practice sentences of all kinds, and rhymes to suit the individual, but always short ones so that there is no effort to remember them.

When the patients have reached a point where they are quite sure of using the right technique and have shaken off wrong habits, allow them to go to teachers of speech or song if they wish to improve further or to learn to address audiences.

The speech therapist must emphasize that'shouting does not make for audibility, but correct projection does and clear articulation is needed for intelligibility. 